\title{
Comparative Proteomic Identification of Protein Disulphide Isomerase A6 Associated with Tert-Butylhydroperoxide- Induced Liver Injury in Rat Hepatocytes
}

\author{
Chien-Heng Shen ${ }^{a, b}$ Shui-Yi Tung ${ }^{a, c}$ Wen-Shih Huang ${ }^{c, d} \quad$ Kam-Fai Lee ${ }^{e}$

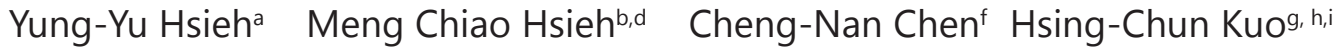 \\ aDepartment of Hepato-Gastroenterology, Chang Gung Memorial Hospital, Chiayi, 'braduate Institute \\ of Clinical Medical Sciences, Chang Gung University, 'Chang Gung University College of Medicine, \\ Taoyuan, dDivision of Colon and Rectal Surgery, Department of Surgery, Chang Gung Memorial \\ Hospital Chiayi, Graduate Institute of Clinical Medical Sciences, College of Medicine, Chang Gung

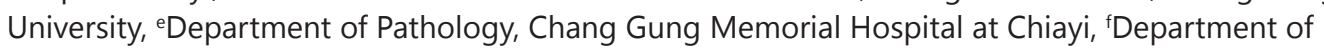 \\ Biochemical Science and Technology, National Chiayi University, Chiayi, 9Department of Nursing, Chang \\ Gung University of Science and Technology, Chronic Diseases and Health Promotion Research Center, \\ CGUST, hResearch Center for Industry of Human Ecology and Research Center for Chinese Herbal \\ Medicine, College of Human Ecology, Chang Gung University of Science and Technology, Taoyuan, \\ 'Research Fellow, Chang Gung Memorial Hospital, Chiayi, Taiwan
}

\section{Key Words}

$\mathrm{T}-\mathrm{BHP} \cdot \mathrm{PDIA6} \cdot \mathrm{ROS} \cdot \mathrm{IRE} 1 \alpha / \mathrm{ASK} 1 \cdot \mathrm{JNK} 1 / 2 \cdot \mathrm{P} 38$

\begin{abstract}
Background/Aims: Oxidants are important human toxicants. They have been implicated in the occurrence and development of liver diseases. Increased intracellular tert-butylhydroperoxide ( $t$-BHP) may be critical for oxidant toxicity, and is commonly used for evaluating mechanisms involving oxidative stress, but the method remains controversial. Methods: Primary cultures of hepatocytes as well as human Hep G2 and mouse FL83B liver cells were obtained. Cell viability was measured by annexin $\mathrm{V}$-FITC/propidium iodide and DAPI staining to determine the effects of $t$-BHP treatment on acute liver injury. A proteomic assay provided information that was used to identify the differentially expressed proteins following $t$-BHP treatment; immunohistochemistry and western blotting were performed to detect the expression of PDIA6 activity in apoptotic and endoplasmic reticulum (ER) stress pathways. Results: Our results demonstrate that $t$-BHP treatment of liver cells increased cell cytotoxicity and the generation of reactive oxygen species. This treatment also increased the level of PDIA6; this was validated in vitro and in vivo based on a comparison of $t$-BHP-treated and -untreated groups. Treatment of mouse liver FL83B cells with $t$-BHP activated caspase 3 , increased the expression of apoptotic molecules, caused cytochrome $\mathrm{c}$ release, and induced $\mathrm{BCl}-2, \mathrm{Bax}$ and IRE1 $\alpha$ /TRAF2 complex formation. $t$-BHP-dependent induction of apoptosis was accompanied
\end{abstract}


Shen et al.: T-BHP Induced an Apoptosis Cascade and ER Stress in Hepatocytes by Upregulation of PDIA6

by sustained phosphorylation of the IRE1 $\alpha / A S K 1 / J N K 1 / 2 / p 38$ pathways and PDIA6 expression. Furthermore, $t$-BHP induced liver FL83B cell viability and apoptosis by upregulating the levels of PDIA6; this process could be involved in the activation of the IRE1 $\alpha / A S K 1 / J N K 1 / 2 / p 38$ signalling pathways. Conclusions: We conclude that $t$-BHP induced an apoptosis cascade and ER stress in hepatocytes by upregulation of PDIA6, providing a new mechanism underlying the effects of $t$-BHP on liver injury.

\section{Introduction}

Reactive oxygen species (ROS) are well recognized as critical signalling molecules; ROS are responsible for cell growth, development, and death in a variety of normal cellular functions $[1,2]$. However, oxidative stress occurs when antioxidant mechanisms are overwhelmed by ROS; the resulting damage can lead to cell death, which can be apoptotic [3, 4]. It has been proposed that with an early increase in intracellular free oxidative stress, ROS can damage cellular components, such as mitochondria, lipids, proteins and DNA, which eventually leads to cell death [5]. Apoptosis is an evolutionarily highly conserved process that induces the degradation of unnecessary or damaged cytoplasmic contents in two cascades, the extrinsic and intrinsic pathways, which both lead to kinase cascades [6]. Apoptosis is characterized by the appearance and activation of TNFR1/Fas in the plasma membrane and by the expression of apoptotic proteins, such as procaspase-3, Bax, and Bcl-2, which translocate to the mitochondria via the kinase signalling cascade, known as the intrinsic pathway [7, 8]. Apoptosis can be activated as an important pathway under numerous extracellular or intracellular stresses, such as starvation and the presence of ROS as well as several prooxidants, such as tert-butylhydroperoxide ( $t$-BHP) [9]. As a short-chain analogue of lipid hydroperoxides, $t$-BHP is widely used in a variety of oxidation processes and is generally employed as a better alternative for ROS, lipid peroxidation, and damage to cell integrity as well as for causing a liver inflammatory reaction [10] in oxidative stress studies. The aim of the present study was to investigate the effect of $t$-BHP on liver cells and to explore the underlying mechanisms of the $t$-BHP target protein.

Hepatocyte apoptosis plays important roles in both the removal of external microorganisms and the occurrence and development of liver diseases, including hepatitis B or C viral infections, excessive fatty liver, fibrosis and drug-induced liver damage $[11,12]$. In a previous study, hepatocyte apoptosis was found to play an important role in liver injury and its regulating factors; determining how hepatocyte apoptosis proceeds is crucial for the treatment of liver diseases [13]. In liver cells, mitochondrial dysfunction resulting from an excess of ROS can alter calcium homeostasis and protein misfolding, while phospholipids can activate endoplasmic reticulum (ER) stress; prolonged ER stress activates Bax and induces apoptosis in addition to the extrinsic and intrinsic pathways [14]. ROS-activated apoptosis signalling kinase 1 (ASK1) resulted in sustained JNK activation, which is a key step in initiating caspase-dependent apoptosis [15]. Recent evidence showed that ROS also contribute to the activation of multiple cellular signalling pathways; for example, IRE1 $\alpha$ binds TNF receptorassociated factor 2 (TRAF2) with ASK1 and further activates Jun N-terminal kinase (JNK) [16]. However, the role of ROS in liver cells treated with $t$-BHP remains unclear.

We recently demonstrated that $t$-BHP plays an essential role in the JNK1/2 and NFKB p50 pathways during cytotoxicity. We also showed that ER stress in rat primary liver cells could signal the discovery of specific hepatitis biomarkers and the mechanism of oxidative agents, including their involvement in hepatotoxicity and redox imbalance involving the ER stress signalling pathway [17]. Moreover, to identify the target of $t$-BHP action on the proteins implicated in ROS metabolism and apoptosis, $t$-BHP-induced changes in rat liver cell protein profiles were investigated in the present study using proteomics approaches (two-dimensional gel electrophoresis-tandem mass spectrometry). In the present study, using proteomics approaches, we also found that $t$-BHP administration could induce the expression of protein disulphide-isomerase A6 (PDIA6), as well as the production of ROS 
and activation of IRE1-alpha/TRAF2, ASK1, JNK1/2, and p38, which could be attributed to the induction of cell death signalling pathways, such as Bcl-2, Bax, and caspase 3, in liver cells.

\section{Materials and Methods}

\section{Cell culture}

Human Hep G2 and mouse FL83B liver cells were obtained from the American Tissue Culture Collection (ATCC, USA). The cells were cultured in Dulbecco's modified Eagle's medium (DMEM) supplemented with $10 \%$ foetal calf serum (Gibco), non-essential amino acids, $1 \mathrm{mM}$ sodium pyruvate, and $1 \%$ antibiotics (100 units/mL penicillin and $100 \mu \mathrm{g} / \mathrm{mL}$ streptomycin). All experiments were performed in plastic tissue culture flasks, dishes, or microplates (Nunc, Naperville, Denmark). Incubation was carried out at $37^{\circ} \mathrm{C}$ in a humidified atmosphere of $5 \% \mathrm{CO}_{2}$ and $95 \%$ air, and the cells were treated with $t$-BHP for $24 \mathrm{~h}$. The cells were collected and washed with phosphate buffered saline and subsequently transferred to sterile plastic tubes for storage at $-80^{\circ} \mathrm{C}$ until use [17].

\section{Primary culture of hepatocytes}

A collagenase perfusion technique [17] was used to isolate hepatocytes from adult 8-week-old male Wistar rats weighing 200-250 g (obtained from National Laboratory Animal Center, Taiwan). The hepatocytes were prepared using two-stage collagenase perfusion, as previously described. The hepatocytes were maintained at a cell density of $2 \times 10^{5}$ cells $/ \mathrm{mL}$ in William's E medium supplemented with $10 \%$ foetal calf serum, an antibiotic mixture, and glutamine. The cells were seeded at a density of $3 \times 10^{4}$ cells in a $0.1 \%$ collagen precoated 96-well plate and were used for the $t$-BHP exposure experiments after being cultured overnight.

\section{Chemical reagents and antibodies}

$t$-BHP was purchased from Sigma (St. Louis, MO, USA) and dissolved in sterile dimethyl sulfoxide or water. It was then stored in aliquots at $-20^{\circ} \mathrm{C}$. Chemical inhibitors for JNK (SP600125), mTOR (rapamycin), p38 (SB203580), and MEK (PD98059), as well as scavengers of ROS (N-acetylcysteine, NAC), were purchased from Sigma Chemical Co. and dissolved as recommended by the manufacturer. 2, 7-dichlorodihydrofluorescein diacetate $\left(\mathrm{H}_{2}\right.$ DCFDA) and anti- $\beta$-actin were purchased from Sigma Chemical Co. Anti-phospho-p44/42 MAPK, anti-phospho-JNK1/2, mouse monoclonal antibodies against caspase 3, Bcl-2, Bax, cytochrome c, caspase 3, cytochrome c, anti-PDIA6, and horseradish peroxidase-linked anti-rabbit or anti-mouse IgG were obtained from Cell Signaling Technology, Inc. (Beverly, MA, USA). Rabbit polyclonal antibodies against ERK Thr ${ }^{202} \mathrm{Tyr}^{204}$, p38 $\mathrm{Thr}^{180} \mathrm{Tyr}^{182}$, JNK $\mathrm{Thr}^{183} \mathrm{Tyr}^{185}$, and ASK1 $\mathrm{Thr}^{845}$ were purchased from Cell Signaling Technology. Inositol-requiring kinase $1\left(\right.$ IRE1 $\alpha \mathrm{Ser}^{724}$ ) and TRAF2 were obtained from Santa Cruz Biotechnology (Santa Cruz, CA, USA). Sodium dodecyl sulphate (SDS), NP-40, sodium deoxycholate, and the protease inhibitor cocktails were purchased from Sigma Chemical Co. TdT-mediated dUTP nick end labelling (TUNEL) kits were purchased from Roche (Germany).

\section{Animal experiments and $t$-BHP treatment}

Adult 8-week-old male Sprague-Dawley rats weighing $200 \pm 50$ g were obtained from the National Laboratory Animal Center, Taiwan. The rats were maintained under specific pathogen-free conditions $\left(22 \pm 0.5^{\circ} \mathrm{C}\right.$ room temperature, $50-60 \%$ relative humidity, $12 \mathrm{~h}$ dark-light alternation with $12-14$ air changes/h) and were supplied with sterilized food and water (Laboratory Rodent Diet 5001, PMI Nutrition International LLC, MO, USA). All experimental protocols involving animals were reviewed and approved by the Institutional Animal Care and Use Committee of Chang Gung University of Science and Technology. All animals were provided with $100 \mathrm{ppm} t$-BHP ad libitum in drinking water for 2 weeks to induce acute liver injury, as described in detail previously [17]. Blood samples were collected through the left femoral vein and immediately stored in heparin-coated capillary tubes for liver enzyme profiling and biochemical analysis, and liver tissue was collected and washed with normal saline and then fixed in $10 \%$ neutral buffered formalin for histopathological evaluation [18]. 


\section{Cellular Physiology Cell Physiol Biochem 2018;45:1915-1926 \\ \begin{tabular}{l|l|l} 
and Biochemistry & $\begin{array}{l}\text { DOI: 10.1159/000487968 } \\
\text { Published online: March 08, } 2018\end{array}$ & $\begin{array}{l}\text { C) } 2018 \text { The Author(s). Published by S. Karger AG, Basel } \\
\text { www.karger.com/cpb }\end{array}$
\end{tabular}}

Shen et al.: T-BHP Induced an Apoptosis Cascade and ER Stress in Hepatocytes by Upregulation of PDIA6

\section{Histopathological evaluation}

Liver tissues were fixed in $10 \%$ buffered formalin embedded in paraffin. For haematoxylin and eosin staining, the tissue samples were cut into 4 - $\mu$ m-thick slides transversely from portal zone 1 to the zone 3 area close to the terminal of the hepatic venule tract, as described in detail previously. Histopathological changes in liver cell morphology were examined by light microscopy (Olympus, Japan) at high power (200x magnification) for each slide. For quantitative purposes, we randomly chose two portal zones in each slide; we used the Image-Pro Plus medical image analysis system to collect photomicrographs, and cell numbers were recorded. Normal hepatocytes were counted from 10 fields randomly chosen in liver samples from every group, using a $200 \times$ magnification light microscope. The mean values for normal cells were calculated per microscope field from six animals in each group [19].

\section{Immunohistochemistry assay (IHC)}

IHC staining was performed using a biotinylated secondary antibody (Vectastain Universal Elite ABC Kit). The monoclonal rabbit antibodies were diluted in a ratio of 1:100. The omission of primary antibodies was used as the negative control. The slides were washed, dehydrated, and mounted for microscopic examination. Quantitative analysis was performed after IHC staining of nitro-tyrosine, p-JNK, and PDIA6. The average integrated optical density (AIOD) of the positively stained area was evaluated from three randomly selected observation fields in each liver section. The data are expressed as the mean \pm SD $(n=$ 6 per group; $\mathrm{p}<0.05$, compared with the control group). The experiments were performed in triplicate and independently, and the data are presented as three repeats from one independent experiment. The immunoreactive cells (brown) were counted using an Olympus Cx31 microscope with the Image-Pro Plus medical image analysis system. The positive area and optical density (OD) of positive cells were determined by measuring three randomly selected microscopic fields (200× magnification) for each slide. The IHC index was defined as the AIOD (AIOD = positive area $\times$ OD/total area) [20].

\section{Two-dimensional differential gel electrophoresis and image analysis}

Total proteins were extracted from $t$-BHP-treated and -untreated cells by incubation with lysis buffer. The protein pellets were re-solubilized in rehydration solution and kept at $-80^{\circ} \mathrm{C}$ until further analysis. The chemicals and reagents used for two-dimensional gel electrophoresis have been previously described [21]. The total amount of protein was determined using the Bradford assay with bovine serum albumin as the standard sample for normalization; following cell lysis, the total cell protein was precipitated with $10 \%$ trichloroacetate in acetone. The protein samples were suspended in rehydration solution and subjected to isoelectric focusing in 13-cm immobilized ReadyStrip ${ }^{\text {TM }}$ (Bio-Rad, Hercules, CA) IPG Strips (pH 3-10) in an Ettan IPGphor II apparatus (Amersham Biosciences). The second dimension of electrophoresis was carried out using 10\% SDS-polyacrylamide gel electrophoresis (PAGE) gels. Then, BioSafe Coomassie Stain was added, and the gels were stained overnight.

In-gel digestion and identification of peptide fingerprints using matrix-assisted laser desorption ionization-time-of-flight/time-of-flight (MALDI-TOF/TOF)

$t$-BHP-treated and -untreated hepatocytes $\left(4 \times 10^{6}\right.$ cells $)$ were harvested for total protein extraction using cell lysis solution (PRO-PREP, Intron Inc., Seoul, Korea). Six pairs of silver-stained two-dimensional SDS-PAGE gels in which total cell proteins had been resolved were scanned using ImageMaster 2D Platinum Software 6.0 (Amersham Biosciences). The software was used for spot detection and matching of each pair of silver-stained gels that differed by at least 3-fold, as described in our previous study [22]. The quantity of each spot was recorded and compared, and the protein spots that were subjected to in-gel digestion for MALDI-TOF/TOF mass spectrometric analysis with a matrix-assisted laser desorption/ionizationliquid chromatography-electrospray ionization (MALDI-LC-ESI) configuration. The gel pieces were then dehydrated and subjected to trypsin digestion. Mass spectra were acquired as the sum of the ion signals, and the peptide fingerprints were selected in the mass range of 700-4000 Daltons and then analysed by the Mascot search engine (http://www.matrixscience.com). The search parameters were defined as follows: database, Swiss-Prot; taxonomy, Homo sapiens; enzyme, trypsin. A Mascot score with p $<0.05$ was considered significant, as previously described. MALDI-TOF/TOF data were searched using in-house Mascot software (ver. 2.2.04). Protein identification required detection of unique peptides, and proteins with more than two spectral counts were selected for further analysis based on their molecular mass and 
photoionization values. Proteins identified with a higher Mascot score in the bovine database than in the human database were considered to be serum contamination and removed [21, 22].

\section{Measurement of cell viability and ROS}

Cell viability, as previously reported using Annexin V-FITC/propidium iodide staining (Biosource International, USA), was used to quantify the percentage of cells undergoing apoptosis. The intracellular accumulation of ROS (O2-) was determined using the fluorescent probe $\mathrm{H}_{2}$ DCFDA. The cells were washed prior to FACS analysis with the Cell Quest software (Becton Dickenson), as previously described [19]. The results are presented as a percentage of the fluorescent intensity compared with the control sample. The apoptotic cells (V+/PI-) were measured by fluorescence-activated cell sorting in a FACS analyser (Becton Dickinson). The data represent three independent experiments.

\section{Preparation of total cell extracts and immunoblot analyses}

The cells were lysed by RIPA lysis buffer (50 mM Tris- $\mathrm{HCl}(\mathrm{pH} 7.4), 1 \% \mathrm{NP}-40,0.25 \%$ sodium deoxycholate, $150 \mathrm{mM} \mathrm{NaCl}, 1 \mathrm{mM}$ EDTA, $1 \mathrm{mM}$ PMSF, $1 \mathrm{mg} / \mathrm{mL}$ aprotinin, $1 \mathrm{mM} \mathrm{Na} \mathrm{VO}_{4}$, and $1 \mathrm{mM} \mathrm{NaF}$ ). The total cell lysate (50 $\mu$ g protein) was separated by SDS-PAGE (12\% running, 4\% stacking) and analysed using the designated antibodies and the western-light chemiluminescent detection system (Bio-Rad, Hercules, CA), as previously described [23].

\section{Statistical analysis}

Data were reported as the mean \pm SD of three independent experiments and were analysed by one-way analysis of variance. The data were analysed using the SAS statistical package "SigmaPlot" version 9.0 (SAS Institute Inc., Cary, NC, USA) [21].

\section{Results}

Two-dimensional profiling of the differentially expressed proteins of primary rat hepatocytes treated with $t$-BHP

Several studies have linked ER dysfunction and activation of the unfolded protein response (UPR), including inositol-requiring ER-to-nucleus signalling protein 1 (IRE1); the UPR is also positively correlated with liver disease severity [24]. Our previous data demonstrated that exposure of rat-derived liver cells to $1.5 \mathrm{mM} t$-BHP for $24 \mathrm{~h}$ triggers ER stress and cell death in multiple cell types [17]. To determine the molecular mechanisms linking $t$-BHP and cell death in primary rat hepatocytes, proteomic technologies can be used to screen the target molecules of $t$-BHP activities. These targets can be identified as novel proteins in oxidative expression profiles and can elucidate the mechanisms underlying activation of the ER stress cascades. In our proteomic study of rat-derived liver cells, the lysates were prepared, and then the protein spots were visualized. Fig. 1A illustrates the selected gel region and the quantification of these two-dimensional spots observed after $t$-BHP treatment in the silver-stained 2D-PAGE analysis. For the image analysis, protein expression profile gels (six pairs from the control and the $t$-BHP-treated groups) were compared using image analysis (ImageMaster software). The pI and MW values of the gel spot were 4.8 and $48 \mathrm{KD}$, respectively, and the spot was confirmed to be PDIA6 by MALDILC-ESI, which implicates this protein in oxidative stress and UPR sensing (IRE1 $\alpha)[25,26]$.

Validation of the differentially displayed proteins in the $t$-BHP-induced liver failure model

Based on these studies, we tested whether $t$-BHP administration caused an oxidative stress-like effect on the biochemical markers of acute hepatitis in rats. $t$-BHP was administered to rats by a predetermined dosage of $t$-BHP ad libitum in drinking water for 2 weeks. As shown in Table 1, $t$-BHP has a pro-inflammatory effect on rat models of liver injury, based on biochemical analyses of the $t$-BHP treatment group compared to the control group [18]. The plasma alanine aminotransferase (ALT) and aspartate aminotransferase (AST) levels were significantly elevated in the $t$-BHP treatment group compared to the group receiving normal saline infusion ( $p<0.05, n=6)$. Serum thioredoxin (TRX) and glutathione (GSH) levels have recently been established as indicators of oxidative stress in various 
Fig. 1. Expression of PDIA6 in t-BHP-treated and control groups in rat livers. (A) The PDIA6 protein spot was subjected to peptide fingerprint identification by MALDITOF/TOF, while the PDIA6 spot was subjected to liquid chromatography-tandem mass spectrometry analysis. The Mascot score of the PDIA6 spot was confirmed by MALDI-TOF-MS. After protein hydrolysis, the number of peptides matching the database was 24 (B, C, D); control rats (I), rats receiving t-BHP infusion (II). The pos-



C
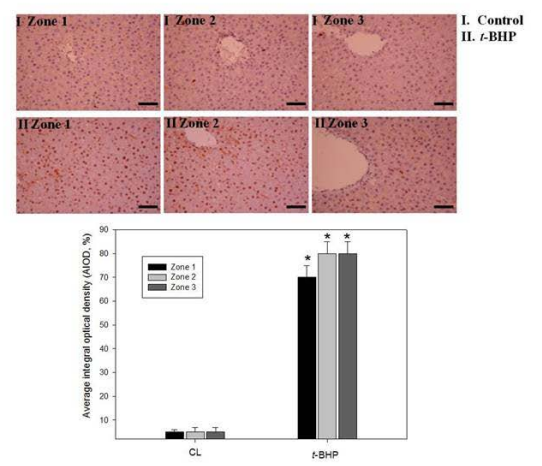

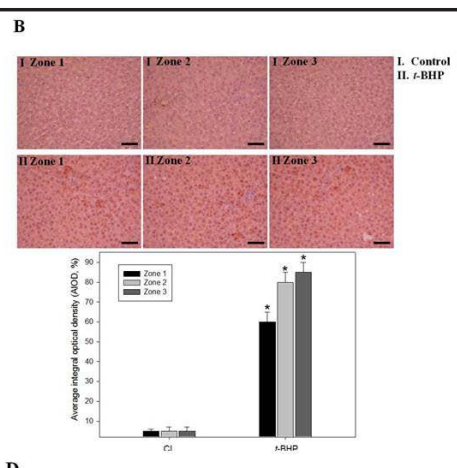

D

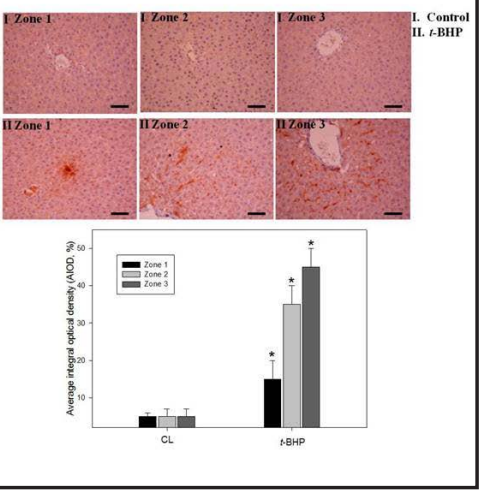
itive-stained area was evaluated from three randomly selected observation fields from each liver section. Quantitation of nitrotyrosine, p-JNK, and PDIA6 was performed using average integrated optical density (AIOD). The data are expressed as the mean $\pm \mathrm{SD}\left(\mathrm{n}=6\right.$ /group). ${ }^{*} \mathrm{p}<0.05$, compared with the control group.

diseases. The administration of $t$-BHP alone significantly depleted $(\mathrm{p}<0.01)$ GSH levels by $80 \%$ and serum TRX levels by $60 \%$. In addition, $t$-BHP administration was found to be a result of excessive ROS production or a consequence of hepatitis, which has been reported to be highly upregulated in response to protein biomarkers of reactive nitrogen species, nitrotyrosine, and sustained JNK1/2 activation (Figs. 1B and 1C). We validated the presence of PDIA6 protein in the $t$-BHP rats, which was consistent with the two-dimensional gel electrophoresis results; $t$-BHP markedly induced PDIA6 compared with the control group, as shown in Fig. 1D. A quantitative examination of hepatocyte pathology in liver tissues from zones 1,2 , and 3 by histopathological evaluation showed that the number of normal hepatocytes present in the $t$-BHP infusion group was significantly lower than the number in the control group (Fig. 2A). The TUNEL assay results are shown in Fig. 2B. Global IHC staining for apoptosis detection was localized primarily within liver tissues in $t$-BHP-treated rats; there was also an increase in the number of TUNEL-positive cells, a marker of apoptosis, in the liver zones 1, 2, and 3 when the $t$-BHP-treated group was compared with the untreated control rats $(t$-BHP: $10 \pm 4,15 \pm 5$, and $42 \pm 3$; control: $2 \pm 1,2 \pm 1$, and $2 \pm 2$; $\mathrm{p}^{*}<0.05, \mathrm{n}=6$ ). For a positive control, DNase (labelled "nuclease" in the kit) was added to the TdT reaction; for a negative control, TdT enzyme was omitted from the reaction. From our results in the same liver zones 1,2 , and 3 with or without $t$-BHP. The positive control (DNase added) should exhibit a large TUNEL-positive signal, distributed uniformly across all tissues in the respective section.
Table 1. Mean serum levels of liver enzymes, Glutathione and thioredoxin in t-BHP-treated rats

\begin{tabular}{lcc}
\hline & Control $(\mathrm{n}=6)$ & $\mathrm{t}$-BHP $(\mathrm{n}=6)$ \\
\hline ALT U/L & $32 \pm 5$ & $82 \pm 9$ \\
AST U/L & $122 \pm 6$ & $395 \pm 15$ \\
$\mathrm{GSH} / \mathrm{mg}$ & $565 \pm 13$ & $111 \pm 10$ \\
$\mathrm{TRX} \mathrm{ng} / \mathrm{ml}$ & $65 \pm 5$ & $28 \pm 7$ \\
\hline
\end{tabular}


Shen et al.: T-BHP Induced an Apoptosis Cascade and ER Stress in Hepatocytes by Upregulation of PDIA6

Fig. 2. Effect of histological changes in the liver of t-BHP-treated rats. The livers were haematoxylin and eosin stained to examine the portal triads (zone 1), zone 2 and the terminal hepatic venules (zone 3). (A) Saline infusion control (I) and t-BHP infusion at indicated dose ad libitum in drinking water for 2 weeks (II). Liver pathology analysis of the control group showed normal hepatocyte structure and arrangement. The t-BHP-treated group demonstrated damaged liver structure. (B) Quantitative evaluation of the pathology analysis. Histological examination of the liver in three zones, as determined by TUNEL IHC staining. Representative liver sections were stained from the control group (I) and rats treated with t-BHP (II); apoptotic cells were measured by microscopy as described in the Materials and Methods section. The t-BHP-treated group exhibited a reduced number of normal hepatocytes, as indicated by the apoptotic cells (filled arrow). Normal hepatocytes were counted from 10 random fields (200x magnification) of each liver sample; the values represent the mean \pm SD of six rats. The data are presented as the mean \pm SD of three independent experiments. ${ }^{*} \mathrm{p}<0.05$, compared with control group.

The negative control should yield a low-intensity background and signal only (Fig. 2B).

t-BHP increased apoptotic body formation, involvement of the ER stress IRE1 $\alpha / A S K 1 / J N K 1 / 2 /$ p38 signalling pathways, and ROS generation in liver cells

To verify that $t$-BHP induced cell toxicity, we also

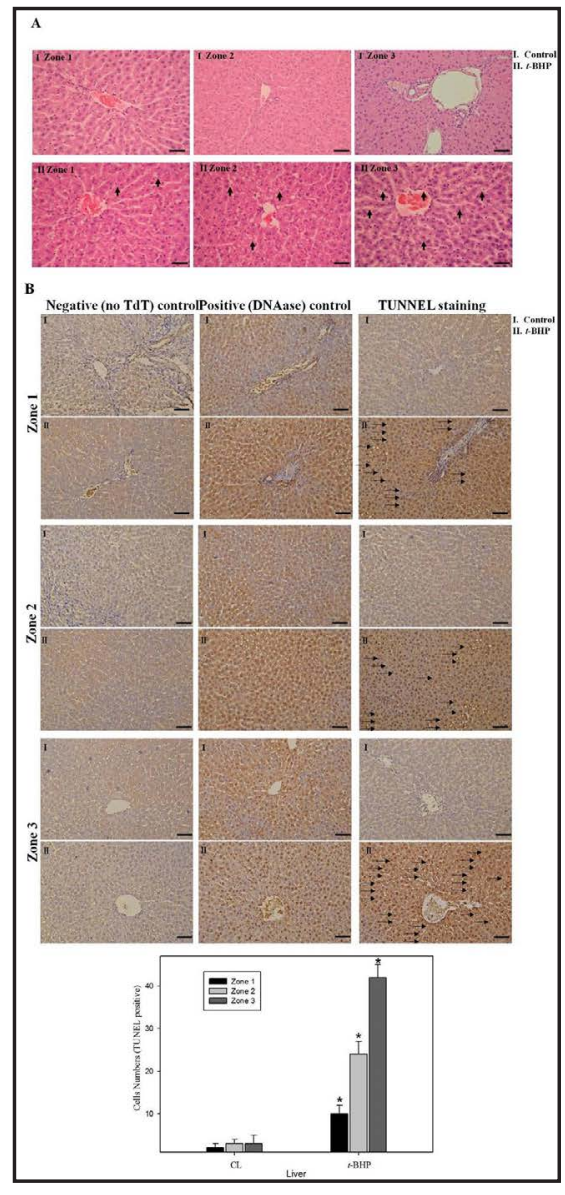
examined the morphological changes in human Hep G2 and mouse FL83B liver cells. As shown in Figs. 3A and 3B, $t$-BHP induced significant cell death as determined by annexin V-FITC/PI staining. The extent of apoptosis was quantified as the percentage of annexin V-positive cells. The extent of apoptosis was $85 \%$ and $60 \%$ in the treated and untreated groups, respectively, $12 \mathrm{~h}$ after the addition of $1.5 \mathrm{mM} t$-BHP. $t$-BHP initially increased the generation of superoxide in mouse FL83B liver cells after $24 \mathrm{~h}$, causing an induction to $32 \%$ compared with the control group (Fig. 3C), as examined using an H2DCFDA probe [27]. Based on this concentration range, we investigated whether $t$-BHP could induce apoptosis and liver injury, potentially involving the ER stress signalling pathway. We investigated whether $t$-BHP produces substantial ER stress leading to apoptosis in mouse FL83B liver cells. To determine the effects of $t$-BHP-induced apoptosis on the persistent extrinsic apoptosis pathway via activation of the caspase cascade in FL83B liver cells, we used kinetic studies to evaluate caspase 3, Bax, and Bcl-2 expression, as well as PDIA6 expression, during a 24-h period. As shown in Fig. 4A, t-BHP increased the levels of Bax to a detectable level and caused cytochrome $c$ release in the cytosol. Additionally, time-dependent cleavage of Bcl-2 for $24 \mathrm{~h}$ and treatment with $t$-BHP resulted in proteolytic processing of caspase 3 (17-36 kDa) into several smaller subunits. Additionally, it was shown that $t$-BHP induces the association of TRAF2 with IRE1 $\alpha$ constituting hallmarks and phospho-IRE1 $\alpha$ Ser724 for the ER stress response. $t$-BHP also increased the expression of PDIA6, which was consistent with the 2-DE results. This finding, presented in Fig. 4B, shows significantly increased levels of total and phosphorylated ASK1, p38 and JNK1/2, compared with the control after $6 \mathrm{~h}$ of exposure to the indicated $t$-BHP dose. This result shows that it is often accompanied by activation of ER stress-mediated TRAF2/IRE1 $\alpha /$ ASK1/p38/JNK as a mechanism that induces cell death and excessive oxidative stress in mouse FL83B liver cells. $t$-BHP-induced apoptosis was accompanied by sustained phosphorylation of the IRE1 $\alpha /$ ASK1/JNK1/2/p38 
Fig. 3. Effects of t-BHP on assessment of cell death in human hepatoma G2 and mouse liver FL83B cells. (A) After treatment for $24 \mathrm{~h}$, the cells were stained with FITC-conjugated Annexin-V and PI for flow cytometry analysis, as described in the Materials and Methods section. The percentages presented in each frame denote the apoptotic cells. (B) Nuclear changes shown by DAPI staining. The liver cells were treated with t-BHP and stained with DAPI. Apoptotic cells were measured using fluorescence microscopy as pre-


viously described. The apoptotic cells are indicated by condensed and damaged nuclei (red arrows). Magnification $\times 200$. (C) The liver cells were treated with t-BHP for $24 \mathrm{~h}$, and intracellular ROS levels were determined by FACS analysis, as described in the Materials and Methods section. Representative histograms show typical FACS profiles for $\mathrm{H}_{2}$ DCFDA in untreated or t-BHP-treated cells. The data are presented as the mean \pm SD based on three independent experiments.

Fig. 4. t-BHP induced cell death via Bax, cytochrome c, caspase 3 , and activated ER stress-associated ASK1/p38/JNK signalling pathways in mouse liver FL83B cells. The data presented in the western blots are derived from a representative study, and comparisons of protein expression are calculated from three replicate experiments. The association of TRAF2 with IRE1 $\alpha$ was determined by immunoprecipitation with TRAF2, followed by western blotting with an anti-IRE $1 \alpha$ antibody.

downstream signalling pathways, and we attempted to identify the downstream target for $t$-BHP. The results showed the effect of

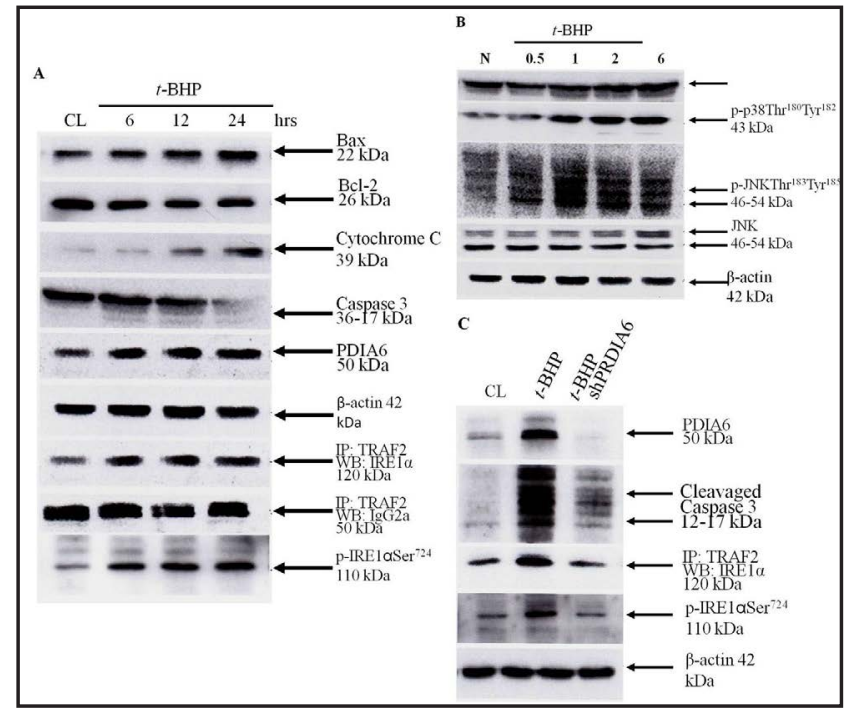
addition of $t$-BHP and lenti shRNA

PDIA6 and confirmed the hypothesis that UPR-associated increases in chaperone PDIA6 expression, induced by $t$-BHP in mouse liver FL83B cells, are mediated via the IRE1alpha signalling pathways. The results in Fig. 4C show that the addition of $t$-BHP and lenti shRNA PDIA6 in mouse FL83B liver cells almost blocked the $t$-BHP-induced expression of PDIA6, the proteolytic processing of caspase 3 and the phosphorylation of IRE $1 \alpha$ as well as the association of TRAF2 with IRE1 $\alpha$ as compared with $t$-BHP treatment at $6 \mathrm{~h}$.

ROS as well as the IRE1 $\alpha / A S K 1 / J N K 1 / 2$ and p38 signalling pathways contribute to the tBHP-induced increase in PDIA6, cell death, and oxidative stress in liver cells

Previous studies have demonstrated $t$-BHP-mediated apoptosis and protein folding involved in the ER stress signalling pathway. In addition, recent reports have shown that several partners are able to phosphorylate or activate TRAF2/IRE1 $\alpha /$ ASK1/p38/JNK and have been associated with toxicity in hepatocytes. We detected the protein PDIA6 referring to 
Shen et al.: T-BHP Induced an Apoptosis Cascade and ER Stress in Hepatocytes by Upregulation of PDIA6

Fig. 5. Effects of kinase inhibitors on the t-BHP-stimulated PDIA6 response to ER stress with sustained phosphorylation of IRE1, JNK1/2 and p38, associated with expression of CCAAT-enhancer-binding protein homologous protein. The kinase inhibitors were treated with or without OGD for $1 \mathrm{~h}$. Total cell lysates were prepared and subjected to western blotting analysis. The protein levels of phosphorylated JNK1/2 and p38 MAPK and the association of TRAF2 with IRE1-alpha and $\beta$-actin were detected with the indicated antibodies. ${ }^{*} \mathrm{p}<0.05$, compared with the control group.

accumulation at the ER sensor, which previously had been identified as the production of ROS, triggering apoptosis. To confirm the association between $t$-BHP action in the differential expression proteins of PDIA6 and activation of the IRE1 $\alpha /$ ASK1/JNK1/2 and p38 signalling pathways contributing to cell death or injury,

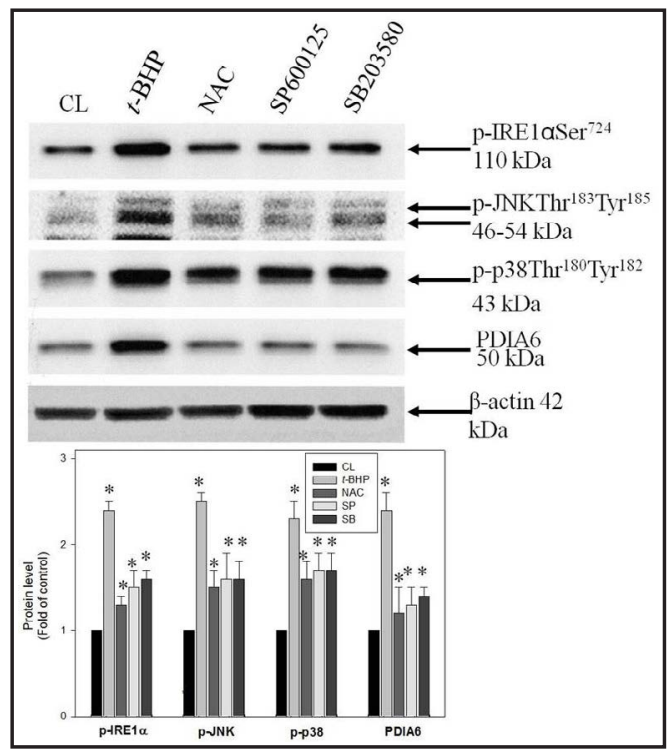
we investigated whether the effects of kinase inhibitors reversed $t$-BHP-induced ER stress signalling. As shown in Fig. 5, mouse FL83B liver cells were subjected to $t$-BHP and then co-treated with the ROS scavenger NAC, the JNK1/2 MAPK inhibitor SP600125, and the p38 MAPK inhibitor SB203580. NAC, SP600125, and SB203580 almost completely blocked the $t$-BHP-induced expression of PDIA6, as well as the phosphorylation of IRE1 $\alpha /$ ASK1/ JNK1/2 and the p38 signalling pathway, compared with $t$-BHP treatment at $6 \mathrm{~h}$. Differentially displayed proteins were assayed from mouse FL83B liver cells and validated; the effects of $t$-BHP-induced apoptosis and ER stress-mediated TRAF2/IRE1 $\alpha /$ ASK1/p38/JNK pathways in liver cells were detected through ROS triggering that resulted in downstream PDIA6 expression.

\section{Discussion}

Hepatocytes have a strong ability to regenerate when facing damaging targets, including ischaemia, hepatitis B or C virus infection, alcohol, cholestatic disorders, drug-induced liver damage, and many liver diseases involving apoptosis by massive ER stress [28, 29]. Several mediators are implicated in ER stress-associated cell death, namely, the oxidative stress that results from the overproduction of ROS and reactive nitrogen species detoxification; superoxide radical anion can effectively interact with antioxidant molecules, which might serve as a common mechanism [30-33]. In this study, the cytotoxic effect of $t$-BHP on liver cells was investigated. Elevation of the inflammatory and oxidative markers ALT and AST, as well as GSH and TRX in rat serum, were observed after $t$-BHP administration. Figs. 1 and 2 also show apoptosis in the liver tissues of the $t$-BHP treatment group; nitro-tyrosine and phosphorylation of JNK1/2 increased after $t$-BHP administration, as mediators of $t$-BHPinduced liver injury. We also found that $t$-BHP induced human hepG2 cells and mouse FL83B liver cell accumulation in the time-dependent induction of apoptosis (Fig. 3), similar to previous results from human Chang liver cells (unpublished data). Treatment with $t$-BHP triggers apoptosis through a step that involves the generation of ROS and cytochrome c release from mitochondria, resulting in the activation of Bax and caspase 3 and a decrease in the cellular levels of Bcl-2 in FL83B liver cells. Moreover, $t$-BHP-induced liver cell apoptosis led to IRE1 $\alpha$ activation and recruitment of the adaptor protein TRAF2, with subsequent activation of apoptosis signal-regulating kinase 1 (ASK1), p38, and JNK1/2 (Fig. 4). Previous studies have shown that p38 and JNK1/2 have several proapoptotic effects, including phosphorylation-induced activation of the proapoptotic Bax and inactivation of the antiapoptotic Bcl2 protein [34]. ASK1 is also a key mediator of ER stress-induced activation downstream of IRE1 $\alpha$ [35]. 
Although studies have implicated IRE1 $\alpha$ in regulating ER stress-induced apoptosis, it is unclear in which context and by what mechanism IRE1 $\alpha$ mediates downstream versus liver death. To identify hepatocyte biomarkers, comprehensive proteomic analyses may identify the PDIA6-mediated mechanisms underlying the oxidative properties of $t$-BHP in pathological processes; PDIA6 was abundant in vivo and in vitro (Fig. 1) [36]. Our results in $t$-BHP-treated hepatocytes showed peroxidative alterations related to ER stress signalling events and ROS overload in the mitochondria. The activation of ROS production and IRE1 $\alpha$ / ASK1/p38/JNK by $t$-BHP was linked to cell death. Additionally, we suggest that $t$-BHP-induced cell viability and activation of IRE1 $\alpha /$ ASK1/p38/JNK decrease could change remarkably after pretreatment with NAC, SP60015, and SB203580 kinase inhibitors. We suggest a necessary role for the IRE1 $\alpha /$ ASK1/p38/JNK pathways and a downstream target of PDIA6 expression during the triggering of liver cell apoptosis and oxidative injury (Fig. 5). Many studies have suggested that PDIA6 overexpression, which is found abundantly in rat liver, has a role in essential cellular processes, such as the redox chaperone function of the ER, and serves as a vital cellular defence against general protein misfolding via its chaperone activity, apoptosis induction, and production of ROS in oxidation, with redox behaviour in various diseases [37, 38]. In a previous study, various changes in the cellular environment were found to trigger the UPR, from oxidative changes to the accumulation of misfolded proteins in the ER, resulting in a UPR sensor; a luminal enzyme, PDIA6, controls the duration of IRE1 activity [39, 40]. Our recent studies on the ER stress response provided new insights into liver cell death induced by $t$-BHP; these results showed that $t$-BHP inhibits rat liver by activating the IRE1 $\alpha / A S K 1 / \mathrm{p} 38 /$ JNK pathway, which is preceded by the association of IRE1 $\alpha /$ TRAF2 via downstream PDIA6 (Fig. 6). However, further studies to reduce the levels of aberrantly modified PDIA6 might also be necessary in liver disease to defend against the pro-apoptotic properties of PDIA6. At the cellular level, there are important unanswered questions that need to be addressed before the therapeutic applications of PDIA6 can be realized [41].

\section{Conclusion}

Based on proteomic differential protein analysis, we demonstrated a new mechanism for liver damage via $t$-BHP-induced cell death; the UPR and oxidative stress correspond to the effects of PDIA6 accumulation at ER-mitochondrial locations, thereby triggering apoptosis. This apoptosis is controlled by ROS and the IRE1 $\alpha /$ ASK1/JNK1/2/p38 signalling pathway (Fig. 6). This study provides additional evidence that the possibility of finding novel therapeutic agents targeting ER organelle-based ROS for the improvement of hepatocyte apoptosis would play an important role in liver injury diseases.

\section{Acknowledgements}

Funding for this study was provided in part by research grants from the Tomorrow Medical Foundation, Taiwan. This study was supported by grants BMRPD42, CLRPG8G0591, CMRPG6B0271, CMRPG6B0272, CMRPF6F0032, CMRPF6G0011, CMRPF6G0012 from Chang Gung Memorial Hospital, Chiayi, Taiwan, and

Fig. 6. Schematic presentation of the signalling pathways involved in t-BHP-implicated death of hepatocytes. The effect of t-BHP on the production of ROSactivated phosphorylation or phosphorylation of IRE1 $\alpha /$ ASK1/ JNK1/2/p38 pathways and PDIA6 expression, which induced PDIA6 upregulation and trig-

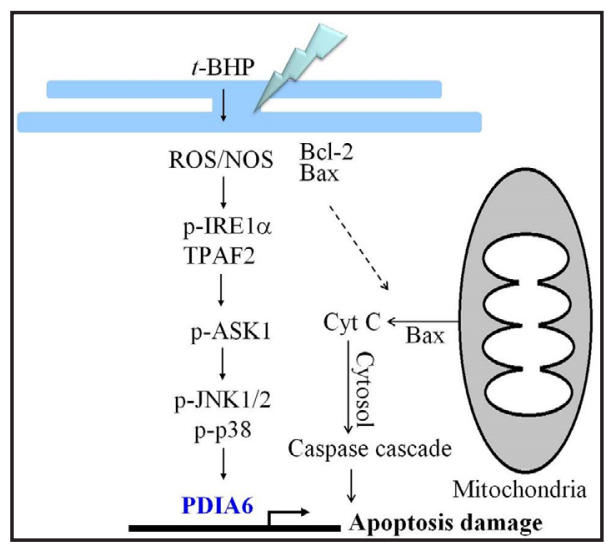
ger an apoptosis cascade, increased the levels of Bax and cytochrome $\mathrm{c}$ release in the cytosol and led to a time-dependent cleavage of Bcl-2. 


\section{Cellular Physiology Cell Physiol Biochem 2018;45:1915-1926 \\ \begin{tabular}{l|l|l} 
and Biochemistry Published onlIne: March 08, 2018 & $\begin{array}{l}\text { C) 2018 The Author(s). Published by S. Karger AG, Basel } \\
\text { www.karger.com/cpb }\end{array}$ \\
\hline
\end{tabular}}

Shen et al.: T-BHP Induced an Apoptosis Cascade and ER Stress in Hepatocytes by Upregulation of PDIA6

Chang Gung University of Science and Technology, Chia-Yi Campus, Taiwan and by the Ministry of Science and Technology, Taiwan (MOST 104-2320-B-255 -003 -MY3).

\section{Disclosure Statement}

There is no financial/commercial conflict of interest.

\section{References}

1 Das DK, Maulik N, Engelman RM: Redox regulation of angiotensin II signaling in the heart. J Cell Mol Med 2004;8:144-152.

2 Irani K: Oxidant signaling in vascular cell growth, death, and survival : a review of the roles of reactive oxygen species in smooth muscle and endothelial cell mitogenic and apoptotic signaling. Circ Res 2000;87:179-183.

3 Haidara K, Morel I, Abalea V, Gascon Barre M, Denizeau F: Mechanism of tert-butylhydroperoxide induced apoptosis in rat hepatocytes: involvement of mitochondria and endoplasmic reticulum. Biochim Biophys Acta 2002;1542:173-185.

Malhi H, Kaufman RJ: Endoplasmic reticulum stress in liver disease. J Hepatol 2011;54:795-809.

Panieri E, Santoro MM: ROS signaling and redox biology in endothelial cells. Cell Mol Life Sci 2015;72:3281-3303.

Battaglia V, Compagnone A, Bandino A, Bragadin M, Rossi CA, Zanetti F, Colombatto S, Grillo MA, Toninello A: Cobalt induces oxidative stress in isolated liver mitochondria responsible for permeability transition and intrinsic apoptosis in hepatocyte primary cultures. Int J Biochem Cell Biol 2009;41:586-594. Desagher S, Osen-Sand A, Nichols A, Eskes R, Montessuit S, Lauper S, Maundrell K, Antonsson B, Martinou JC: Bid-induced conformational change of Bax is responsible for mitochondrial cytochrome c release during apoptosis. J Cell Biol 1999;144:891-901. Luo X, Budihardjo I, Zou H, Slaughter C, Wang X: Bid, a Bcl2 interacting protein, mediates cytochrome c release from mitochondria in response to activation of cell surface death receptors. Cell 1998;94:481-490. Frey RS, Ushio-Fukai M, Malik AB: NADPH oxidase-dependent signaling in endothelial cells: role in physiology and pathophysiology. Antioxid Redox Signal 2009;11:791-810. Zhao W, Feng H, Sun W, Liu K, Lu JJ, Chen X: Tert-butyl hydroperoxide (t-BHP) induced apoptosis and necroptosis in endothelial cells: Roles of NOX4 and mitochondrion. Redox Biol 2017;11:524-534.

-11 Guicciardi ME, Gores GJ: Apoptosis as a mechanism for liver disease progression. Semin Liver Dis 2010;30:402-410.

12 Canbay A, Friedman S, Gores GJ: Apoptosis: the nexus of liver injury and fibrosis. Hepatology 2004;39:273278.

13 Roychowdhury S, McCullough RL, Sanz-Garcia C, Saikia P, Alkhouri N, Matloob A, Pollard KA, McMullen MR, Croniger CM, Nagy LE: Receptor interacting protein 3 protects mice from high-fat diet-induced liver injury. Hepatology 2016;64:1518-1533.

14 Riedl SJ, Shi Y: Molecular mechanisms of caspase regulation during apoptosis. Nat Rev Mol Cell Biol 2004;5:897-907.

15 Luo D, He Y, Zhang H, Yu L, Chen H, Xu Z, Tang S, Urano F, Min W: AIP1 is critical in transducing IRE1mediated endoplasmic reticulum stress response. J Biol Chem 2008;283:11905-11912.

16 Zhao J, Jitkaew S, Cai Z, Choksi S, Li Q Luo J, Liu ZG: Mixed lineage kinase domain-like is a key receptor interacting protein 3 downstream component of TNF-induced necrosis. Proc Natl Acad Sci U S A 2012;109:5322-5327.

-17 Shen CH, Tung SY, Huang WS, Lu CC, Lee KC, Hsieh YY, Chang PJ, Liang HF, Chen JH, Lin TH, Hsieh MC, Kuo HC: Exploring the effects of tert-butylhydroperoxide induced liver injury using proteomic approach. Toxicology 2014;316:61-70.

18 Tsai JJ, Kuo HC, Lee KF, Tsai TH: Proteomic analysis of plasma from rats following total parenteral nutrition-induced liver injury. Proteomics 2015;15:3865-3874.

19 Lu CC, Huang WS, Lee KF, Lee KC, Hsieh MC, Huang CY, Lee LY, Lee BO, Teng CC, Shen CH, Tung SY, Kuo HC: Inhibitory effect of Erinacines A on the growth of DLD-1 colorectal cancer cells is induced by generation of reactive oxygen species and activation of p70S6K and p21. J Funct Food 2016;21:474-484. 


\section{Cellular Physiology Cell Physiol Biochem 2018;45:1915-1926 \begin{tabular}{l|l|l|l} 
DOI: 10.1159/000487968 & (c) 2018 The Author(s). Published by S. Karger AG, Basel \\
www
\end{tabular}

Shen et al.: T-BHP Induced an Apoptosis Cascade and ER Stress in Hepatocytes by Upregulation of PDIA6

20 Tsai JJ, Kuo HC, Lee KF, Tsai TH: Glycyrrhizin represses total parenteral nutrition-associated acute liver injury in rats by suppressing endoplasmic reticulum stress. Int J Mol Sci 2013;14:12563-12580.

-21 Teng CC, Kuo HC, Sze CI: Quantitative proteomic analysis of the inhibitory effects of CIL-102 on viability and invasiveness in human glioma cells. Toxicol Appl Pharmacol 2013;272:579-590.

-22 Lee KC, Kuo HC, Shen CH, Lu CC, Huang WS, Hsieh MC, Huang CY, Kuo YH, Hsieh YY, Teng CC, Lee LY, Tung SY: A proteomics approach to identifying novel protein targets involved in erinacine A-mediated inhibition of colorectal cancer cells' aggressiveness. J Cell Mol Med 2017;21:588-599.

23 Huang WS, Hsieh MC, Huang CY, Kuo YH, Tung SY, Shen CH, Hsieh YY, Teng CC, Lee KF, Chen TC, Lee KC, Kuo HC: The Association of CXC Receptor 4 Mediated Signaling Pathway with Oxaliplatin-Resistant Human Colorectal Cancer Cells. PLoS One 2016;11:e0159927.

24 Haidara K, Marion M, Gascon-Barre M, Denizeau F, Averill-Bates DA: Implication of caspases and subcellular compartments in tert-butylhydroperoxide induced apoptosis. Toxicol Appl Pharmacol 2008;229:65-76.

-25 Rutkevich LA, Cohen-Doyle MF, Brockmeier U, Williams DB: Functional relationship between protein disulfide isomerase family members during the oxidative folding of human secretory proteins. Mol Biol Cell 2010;21:3093-3105.

26 Jessop CE, Watkins RH, Simmons JJ, Tasab M, Bulleid NJ: Protein disulphide isomerase family members show distinct substrate specificity: P5 is targeted to BiP client proteins. J Cell Sci 2009;122:4287-4295.

27 Kuo HC, Kuo YR, Lee KF, Hsieh MC, Huang CY, Hsieh YY, Lee KC, Kuo HL, Lee LY, Chen WP, Chen CC, Tung SY. A Comparative Proteomic Analysis of Erinacine A's Inhibition of Gastric Cancer Cell Viability and Invasiveness. Cell Physiol Biochem 2017;43:195-208.

28 Lupberger J, Hildt E: Hepatitis B virus-induced oncogenesis. World J Gastroenterol 2007;13:74-81.

29 Liang C, Wang X, Hu J, Lian X, Zhu T, Zhang H, Gu N1, Li J. PTPRO Promotes Oxidized Low-Density Lipoprotein Induced Oxidative Stress and Cell Apoptosis through Toll-Like Receptor 4/Nuclear Factor $\kappa B$ Pathway. Cell Physiol Biochem 2017;42:495-505.

30 Egnatchik RA, Leamy AK, Noguchi Y, Shiota M, Young JD: Palmitate-induced activation of mitochondrial metabolism promotes oxidative stress and apoptosis in H4IIEC3 rat hepatocytes. Metabolism 2014;63:283-295.

-31 Tang Y, Jia W, Niu X, Wu L, Shen H, Wang L, Qi R, Ling C, Li M. CCL2 is Upregulated by Decreased miR-122 Expression in Iron-Overload-Induced Hepatic Inflammation. Cell Physiol Biochem. 2017;44:870-883.

-32 Wu T, Li J, Li Y, Song H. Antioxidant and Hepatoprotective Effect of Swertiamarin on Carbon TetrachlorideInduced Hepatotoxicity via the Nrf2/HO-1 Pathway. Cell Physiol Biochem. 2017;41:2242-2254.

-33 Wang H, Zhang Y, Bai R, Wang M, Du S. Baicalin Attenuates Alcoholic Liver Injury through Modulation of Hepatic Oxidative Stress, Inflammation and Sonic Hedgehog Pathway in Rats. Cell Physiol Biochem. 2016;39:1129-40.

-34 Lei K, Davis RJ: JNK phosphorylation of Bim-related members of the Bcl2 family induces Bax-dependent apoptosis. Proc Natl Acad Sci U S A 2003;100:2432-2437.

-35 Hu P, Han Z, Couvillon AD, Kaufman RJ, Exton JH: Autocrine tumor necrosis factor alpha links endoplasmic reticulum stress to the membrane death receptor pathway through IRE1alpha-mediated NF-kappaB activation and down-regulation of TRAF2 expression. Mol Cell Biol 2006;26:3071-3084.

36 Lin L, Li H, Lin H, Zhang M, Qu C, Yan L, Yin X, Ni J Application of iTRAQ-Based Quantitative Proteomics Approach to Identify Deregulated Proteins Associated with Liver Toxicity Induced by Polygonum Multiflorum in Rats. Cell Physiol Biochem 2017;43:2102-2116.

-37 Eletto D, Eletto D, Dersh D, Gidalevitz T, Argon Y: Protein disulfide isomerase A6 controls the decay of IRE1alpha signaling via disulfide-dependent association. Mol Cell 2014;53:562-576.

-38 Son Y, Cheong YK, Kim NH, Chung HT, Kang DG, Pae HO: Mitogen-Activated Protein Kinases and Reactive Oxygen Species: How Can ROS Activate MAPK Pathways? J Signal Transduct 2011;2011:792639.

39 Hoffstrom BG, Kaplan A, Letso R, Schmid RS, Turmel GJ, Lo DC, Stockwell BR: Inhibitors of protein disulfide isomerase suppress apoptosis induced by misfolded proteins. Nat Chem Biol 2010;6:900-906.

40 de APAM, Verissimo-Filho S, Guimaraes LL, Silva AC, Takiuti JT, Santos CX, Janiszewski M, Laurindo FR, Lopes LR: Protein disulfide isomerase redox-dependent association with p47(phox): evidence for an organizer role in leukocyte NADPH oxidase activation. J Leukoc Biol 2011;90:799-810.

41 Parakh S, Atkin JD: Novel roles for protein disulphide isomerase in disease states: a double edged sword? Front Cell Dev Biol 2015;3:30. 